\title{
CELEBRATING 15 YEARS OF BUG BREAKFAST
}

\section{Lynne Madden}

Editor

\section{Jeremy McAnulty}

Director, Communicable Diseases

NSW Department of Health

Bug Breakfast is the name given to a monthly series of hour-long breakfast seminars on communicable diseases delivered by the Division of Population Health of the NSW Department of Health. The Centre for Epidemiology and Research and the Centre for Health Protection coordinate the content and delivery of the sessions. First delivered in 1990, the purpose of Bug Breakfast is to keep participants abreast of current issues relating to the management of communicable diseases in NSW. It also forms part of the training for public health officer trainees.

The audience for Bug Breakfast has grown considerably over the past 15 years. Since 1999 the sessions have been made available to rural public health sites across NSW by videoconferencing through the resources of the NSW Telehealth Initiative. Currently 11 sites (see Figure 1) are routinely connected to Bug Breakfast, with the seminar transmitted from the live site at the NSW Department of Health, North Sydney. Over a hundred people regularly participate in each session.
FIGURE 1

\section{VIDEOCONFERENCING SITES ROUTINELY CONNECTED TO BUG BREAKFAST}

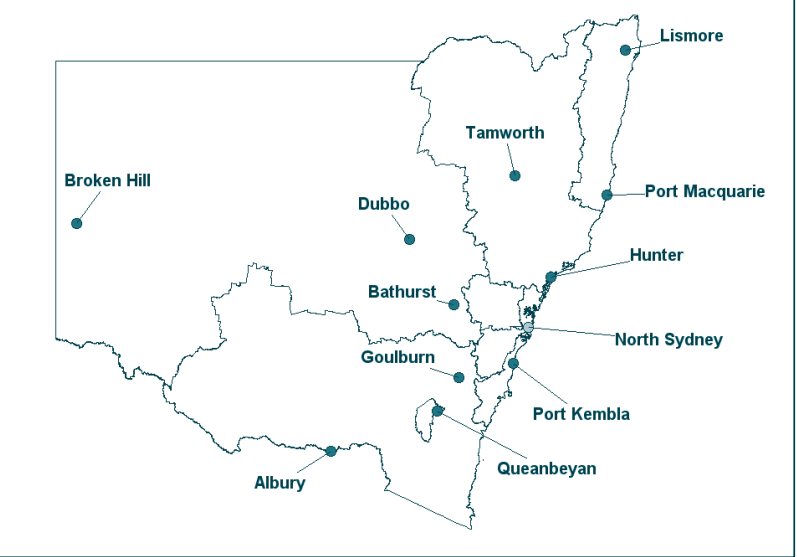

Here we describe two new developments: first, a series of Bug Breakfast web pages for the NSW Health Intranet site and second, a regular feature for the Bulletin, 'Bug Breakfast in the Bulletin', where the content of the recent Bug Breakfast will be summarised.

\section{BUG BREAKFAST ON THE NSW HEALTH INTRANET}

\section{Carlie Naylor}

NSW Public Health Officer Training Program

\section{NSW Department of Health}

To streamline the organisation of Bug Breakfast and ensure that all public health units in NSW have timely access to the information on each session, a series of Bug Breakfast web pages has been developed for the NSW Health Intranet site. Here we describe the content of each of those pages and where they can be accessed.

\section{HOME PAGE}

The Bug Breakfast home page provides a brief description of Bug Breakfast and presents the calendar of dates and session topics for the year. Guidelines have been developed to assist presenters when preparing PowerPoint presentations to be delivered via videoconferencing and these can also be accessed from this page.

\section{PARTICIPANT INFORMATION PAGE}

The participant information page contains a link to the promotional Bug Breakfast flyer, which has a list of locations that are receiving the session and local contact information. Eventually it is anticipated that where speakers make their PowerPoint presentations available these will be loaded onto the website prior to the session. Participants will then be able to access these presentations from this page. Feedback on Bug Breakfast sessions from participants is encouraged and to facilitate this process a Bug Breakfast email address has been established. Participants can click on the link and provide comments and suggestions about the session.

\section{SITE REGISTRATION PAGE}

The site registration web page allows public health units to register their site to participate via videoconference. The site registration form contains several fields, including a dropdown box containing session details, the public health unit name, the facilitator's contact information and the name of the videoconference site for the broadcast. Once the site registration form has been completed and submitted the user receives a message indicating whether they have been successful in registering their unit.

The June 2005 Bug Breakfast was the first session for which the Bug Breakfast web pages were operational. Several public health units accessed the web page, registered their attendance for the videoconference broadcast and provided feedback on the session. 욤 